\title{
EAl Endorsed Transactions

\section{Early and Precise Detection of Pancreatic Tumor by Hybrid Approach with Edge Detection and Artificial Intelligence Techniques}

\author{
Bhawna Dhruv ${ }^{1}$, Neetu Mittal ${ }^{1, *}$ and Megha Modi $^{2}$ \\ ${ }^{1}$ Amity University Uttar Pradesh, Noida, UP, India \\ ${ }^{2}$ Yashoda Super Specialty Hospital, UP, India
}

\section{Abstract}

INTRODUCTION: Pancreatic cancer is highly lethal as it grows, spreads rapidly and difficult to diagnose at its early stages. It can be identified through scan images. The tumorous images obtained from imaging techniques suffer from the drawback of cryptic data due to presence of unwanted noise and poor contrast.

OBJECTIVE: To reduce the risk of pancreatic cancer, its detection and diagnosis at an early stage becomes crucial.

METHODS: The proposed work encompasses the processing of CT scans of pancreatic tumor using classical and artificial intelligence based optimized edge detection techniques for optimization and detection of tumor.

RESULTS: The simulation results are highly encouraging as evident from the far improved visibility of resultant images with Particle Swarm Optimization.

CONCLUSION: The output image with PSO shows the quality enhanced CT images which helps in accurate detection and diagnosis of the pancreatic tumor at an early stage providing an aid in medical imaging.

Keywords: Pancreatic Tumor, Ant Colony Optimization, Genetic Algorithm, Particle Swarm Optimization, Fuzzy C Means Clustering.

Received on 21 April 2021, accepted on 30 May 2021, published on 31 May 2021

Copyright (C) 2021 Bhawna Dhruv et al., licensed to EAI. This is an open access article distributed under the terms of the Creative Commons Attribution license, which permits unlimited use, distribution and reproduction in any medium so long as the original work is properly cited.

doi: 10.4108/eai.31-5-2021.170009

\section{Introduction}

Pancreas is an elongated retroperitoneal organ around 15-20 centimetres in length. Anatomically, it is divided into head, neck, body, tail and uncinate process. The head lies in relation to the second part of duodenum and tail lies in the hilum of spleen. There are two ducts in the pancreas, the main pancreatic duct and the duct of Santorini, known as duct of wirsung and the duct of Santorini respectively. The pancreas secretes both endocrine secretions i.e. by islet cells of langhans- glucagon by alpha cells, somatostatin by delta cells, insulin by beta cells, and pancreatic peptide by pp cells as well as exocrine secretions i.e. pancreatic juice for digestion of proteins, fats and carbohydrates. Needless to say, the pancreas is an inevitable organ which by its gland functions serve multiple physiological roles for disease free survival of human beings. Multiple factors come into play to cause the abuse and disrupt internal milieu of pancreas to lead to pathogenesis of various conditions, some becoming chronic to lead to the evolution of tumors. Notable of these for carcinoma pancreas (exocrine: adenocarcinoma, periampullary carcinoma) are: Smoking, chronic alcoholism, chronic pancreatitis, high fat diet, diabetes mellitus, exposure to carcinogens like benzidine, previous cholecystectomy, obesity, liver cirrhosis, genetic factors like mutation in KRAS, p53 genes, over expression of EFGR, association with other malignancies in syndrome like Peutz Jegher Syndrome, HNPCC. Apart from CA pancreas, there are both exocrine and endocrine tumors of pancreas pertaining to the cells sub serving the corresponding function like (i) Endocrine:

${ }^{2 *}$ Corresponding author. Neetu Mittal Email: nmittal1@amity.edu 
Insulinoma, Gastrinoma, Glucagonoma, VIPoma, Somatostinoma, (ii) Exocrine: Mucinous cystic neoplasms, Serous cystic neoplasm, Intraductal papillary mucinous neoplasm (IPMN).

\section{Related Work}

For effective diagnosis, management and prognosis of various tumors and other medical disorders, modern medical imaging techniques such as X-rays, USG, CT scans, MRI, PET scans are frequently used [1]. The anatomical information provided by these images is invariably important to detect, diagnose, classify and demarcate the extent of abnormalities in the human body [2]. The quality of these images influences the decision making and overall management of the disease both in terms of medical as well as surgical modalities and image guided procedures. The CT is a non-invasive modern imaging technique, highly beneficial for pancreas diagnosis as it provides the detailed anatomical structure of various parts of the organ. However, the CT images are subjected to irregular variations in the human anatomical structures like bony outgrowths, cavitary lesions, vascular areas and soft tissues with varying densities and intensities [3-4]. Such complex medical images with cryptic data and irregular contours are innate in nature and highly tedious to process and interpret [5-7]. Further, the quality of CT images is downgraded due to presence of unwanted noise, poor contrast, weak edges and lack of homogeneity. Thus, there is a need for application of an effective and immaculate computerised image segmentation technique to easily reveal the discrete information from complex medical images.

Image edge detection segmentation is extraction of edges by identifying discontinuities in the intensity of an image and is widely used in computer vision and medical imaging [810]. The classical edge detection technique yields in inferior visibility images with large computation time as every set of operation is carried out for each pixel. The artificial intelligence optimized edge detection segmentation technique may reduce the limitations of classical edge detection techniques and yield in superior quality of resultant images. The most commonly used artificial intelligence optimization techniques include GA, FCM and PSO etc. Fuzzy C means clustering provides precise computation of the cluster membership but has least sensitivity to noise. Alomoush W et al. [11] discussed that the optimum number of clusters in each image should be identified and given by the experts or operators, rendering this approach a semi-automatic method of segmentation that consumes time. Dubey YK et al. [12] concluded that there is a need of improvement in FCM clustering in terms of accuracy, precision and speed. GAs support multi-objective function and utilize transition rules. However, they are known for false convergence or local minima/maxima with large computation time. Several times they are unable to solve restricted optimisation problems effectively with dynamic data sets [13] [14]. Yu M [15] in his study discussed about various factors which affect the efficiency of GA, such as large population size, inefficient fitness function and mismatched crossover technique. Katoch $\mathrm{S}$ et al. [16] described inappropriate population size selection leads to poor quality solution and concluded that if the mutation is not considered during development, no new evolution evidence would be available. ACO is one of the swarm-based algorithms used effectively for combinatorial optimization problems. ACO is a heuristic positive feedback algorithm utilising intelligence of swarms that mimics the ant's behaviour and methods of communication in search of food [17-19]. The major advantages of ACO includes its strong searching capability in a population simultaneously, ability to adapt to new changes and capacity to discover multiple good solutions rapidly. Khanna K et al. [20] explored that adjustment of parameters in ACO totally depend upon the nature of image which emerges as a typical problem in fetching the best solution. Guanyu Wang [21] proposed that ACO is not fit for large size combinatorial problems as it consumes large computational time. The selection of parameters also affects the final outcome. If the pheromone constraints are selected without expertise and theoretical justifications, the results obtained are likely to be greatly dispersed because of the algorithm's inefficiency and poor convergence. Aher et al.[22] proposed a technique for cancer classification using Rider Optimization algorithm and Chicken Swarm Optimization for rapid diagnosis of disease severity evaluation implementing microarray innovation. Microarray innovation is a hybridization based strategy that can gauge the articulation levels of thousands of qualities in a solitary examination. Kaur et al. [23] discussed various data mining and soft computing techniques to prospect diabetes disorders in patients, concluding efficacy of hybrid techniques to be very high and accurate. Sharma et al. [24] presented a review on supervised learning and soft computing techniques used in stress diagnosis of human beings, further discussing their contributions, pros and cons respectively. Sharma et al. [25] discussed the need to investigate opportunity and challenges in the diagnosis of psychiatric disorders using soft computing techniques as $20 \%$ of the youth suffers from one or the other psychiatric disorders as per reports of World Federation of Mental Health in 2018. Sharma et al. [26] proposed a model to examine the performance of swarm intelligence techniques for effective diagnosis of cardiac arrhythmia, concluding satin-bird optimization as highly accurate technique. Singh [27] discussed and reviewed various nature inspired meta heuristic techniques for the better optimization of problems in real world. The PSO is the best-recognized heuristic method based on swarm intelligence, providing efficient solution of various search space optimization problems [28]. It is much faster in computation and requires a smaller set of parameters [29].

In this work, the diagnostic aspects of $10 \mathrm{CT}$ scan images depicting pancreatic tumor of various types have been improved with application of image segmentation techniques. The paper presents computer implementation of four classical edge detection segmentation techniques namely Roberts, Sobel, Prewitt, Canny, four artificial intelligence optimized 
edge detection segmentation techniques (ACO-Prewitt, ACO-Roberts, ACO-Sobel, ACO-Canny), FCM, GA and PSO. Apart from visualization, the computer simulation results have been quantitatively compared in terms of entropy obtained with all the techniques. This paper is organised as section 3 presents methodology, section 4 elaborates results and discussion, conclusion in presented in section 5 .

\section{Methodology}

Segmentation technique is to divide an image into various sections to analyse crucial segments only rather than entire image based on characteristics of the pixels in an image [30]. Various algorithms have been proposed over a period of time to overcome segmentation problems in a specific applications area. These applications may include medical imaging, automated driving and machine vision [31]. The segmentation problem is mathematically given by finding the sets $S_{k} \subset \Omega$. Here, $\Omega$ denotes the domain of an image. Therefore, every set constituting segmentation satisfy equation (1).

$$
\Omega=\prod_{k=1}^{K} S_{k}
$$

where, each $\mathrm{S}_{\mathrm{k}}$ is connected and $\mathrm{S}_{\mathrm{k}} \cap \mathrm{S}_{\mathrm{j}}=\phi$ for $\mathrm{k} \neq \mathrm{j}$.

Image segmentation has a significant role in medical imaging where the scrutiny of delineation in the anatomical structures becomes crucial for better health care. It helps the medical practitioners to identify and diagnose an early-stage illness [32]. Imaging techniques serve as an effective measure for non-invasive diagnosis of a diseases. These advancements have expanded the knowledge of healthy or unhealthy anatomy for medical research and prove to be critical for better diagnosis of a disease [33]. Also, increasing number of medical images has made it important to use computer algorithms such as image segmentation for better analysis [34].

\subsection{Edge Detection Segmentation Techniques}

In order to reduce the unwanted data with preserving crucial properties, edge detection focuses on providing the abstract information of an image [35]. It refers to identifying sharp discontinuities which are in general sudden changes in the pixel intensity leading to formulate boundaries of an object. Discontinuities in the image may arise due to Step Edges (image intensity shifts from one value to other) or Line Edges (intensity differs and bounces back to initial value) [36-37]. However, these are rare conditions and may turn into Ramp Edges and Roof Edges respectively shown in Figure. 1, where the changes in intensity occur instantaneously rather over finite distance.

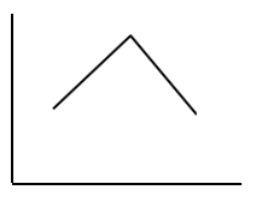

(a) Roof Edge

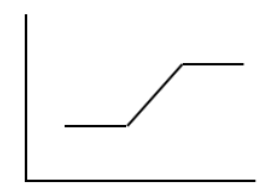

(c) Ramp Edge

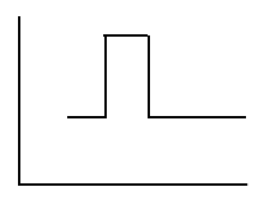

(b) Line Edge

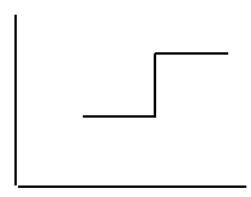

(d) Step Edge
Figure 1. Categories of Edge

The certainty of edge detection algorithms is essential to the overall performance of computer vision and image understanding frameworks. Edge based segmentation, segments the image on the basis of boundaries using the stated algorithm, where the first step is to select the edges of an object using an edge detection operator. Second and third step focusses on computing the gradient amplitude and retaining the edges with respect to threshold [38-39].

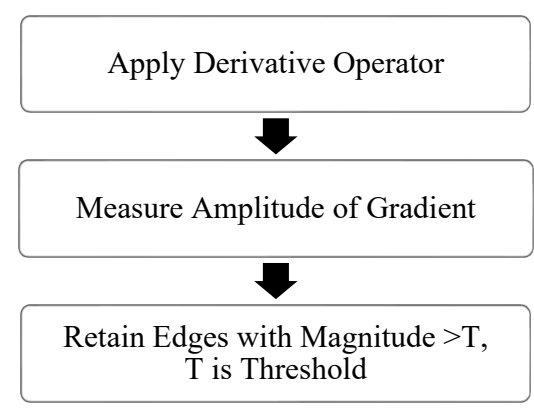

Figure 2. Algorithm of Edge based Segmentation Techniques

Types of Edge Detection Segmentation Techniques To improve the edges in a medical image and perform comparative analysis among classical edge detection segmentation techniques and ACO optimized edge detection segmentation techniques (ACO-Roberts, ACO-Sobel, ACOPrewitt and ACO-Canny) $10 \mathrm{CT}$ scan images have been used.

\section{Roberts}

This operator executes 2D spatial gradient estimation on an image. It uses $2 \times 2$ convolution kernels, where one kernel is 90 degrees rotated around by the other kernel. $G_{x}$ and $G_{y}$ are utilized to estimate the absolute gradient $G$ [40]. Gradient magnitude is computed using equation (2) 
$|f[a, b]-f[a+1, b+1]|-f[a, b+1]+f[a+1, b]=G[f[a, b]]$

With the convolution masks, this equation (3) is

$$
\left|\mathrm{G}_{\mathrm{x}}\right|+\left|\mathrm{G}_{\mathrm{y}}\right|=\mathrm{G}[\mathrm{f}[\mathrm{a}, \mathrm{b}]]
$$

The $G_{x}$ and $G_{y}$ are approximated by-

\begin{tabular}{|c|c|}
\hline+1 & 0 \\
\hline 0 & -1 \\
\hline
\end{tabular}

$\mathrm{G}_{\mathrm{X}}$

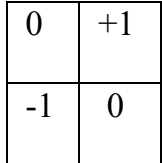

$\mathrm{G}_{\mathrm{y}}$
Sobel

An image implementing sobel operator undergoes 2D spatial gradient measurement and focusses on region having high spatial frequency related to boundaries [41]. The Sobel operator is similar to Roberts and consists of $3 \times 3$ convolution kernel. The second kernel is turned around by 90 degrees of the latter. These kernels are designed so, to receive maximum response at edges running vertically and horizontally and obtain gradient component in both orientations namely Gx and Gy, which are later combined to estimate absolute gradient G. Gradient magnitude can be calculated as equation (4)

$$
\mathrm{M}=\sqrt{S_{x^{2}}+S_{y^{2}}}
$$

The partial derivatives are approximated as given mathematically by equation (5) and (6)

$$
\begin{aligned}
& S_{x}=\left(a_{4}+a_{3}+a_{2}\right)-\left(a_{0}+c a_{1}+a_{6}\right) \\
& S_{y}=\left(a_{2}+c a_{1}+a_{0}\right)-\left(a_{4}+c a_{5}+a_{6}\right)
\end{aligned}
$$

Where $\mathrm{c}$ is a constant equal to 2

\begin{tabular}{|l|l|l|}
\hline-1 & 0 & +1 \\
\hline-2 & 0 & +2 \\
\hline-1 & 0 & +1 \\
\hline
\end{tabular}

$\mathrm{G}_{\mathrm{X}}$

\begin{tabular}{|c|c|c|}
\hline+1 & +2 & +1 \\
\hline 0 & 0 & 0 \\
\hline-1 & -2 & -1 \\
\hline
\end{tabular}

$\mathrm{G}_{\mathrm{y}}$

\section{Prewitt}

Prewitt operator computes the magnitude and detects edges in less computational time using kernel which estimates the magnitude and orientation directly [42]. This operator works on 8 possible orientations in $3 \times 3$ neighbourhood. Out of the 8 convolution masks, one with highest module is then selected.

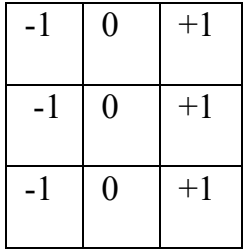

$\mathrm{G}_{\mathrm{x}}$

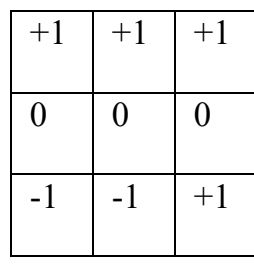

$\mathrm{G}_{\mathrm{y}}$

\section{Canny}

The canny operator recovers the edges by identifying local maxima of gradient in an image and detects both strong and weak edges [43-44]. The weak edges make it to the final image in the event they are affined to the strong edges, hence reducing the chances of presence of noise in the output. The image gradient is computed using gaussian filter derivative [45]. This operator uses the stated algorithm in Figure 3.

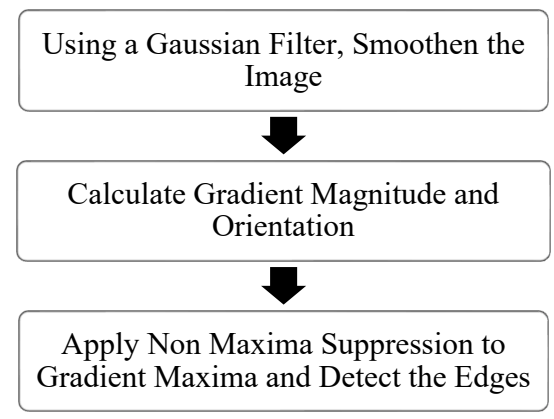

Figure 3. Algorithm of Canny Edge Detection Segmentation Technique

\subsection{Ant Colony Optimization}

Ant colony optimization is a metaheuristic population centred approach to different optimization problems [46]. In ACO, every subject of the total population is a fabricated agent who frames an inherent solution to the concerned problem. The problem is represented graphically by these agents to propose arrangements [47]. At every step, the solution elements are combined to proposed solution defined by their move. The graph which is used to bias the choice of artificial agent and probabilistic model are correlated [48-50]. This model is updated at each step, in order to increase the possibility that upcoming agents will propose a better solution. ACO has been named on based on the activities of real ant species, who determine the minimum path from a location to a origin of food by manipulating the pheromone which is deposited on ground while moving. The artificial ants also work in similar way by depositing the artificial pheromone on the graph based on problem they intend to solve [51]. However, it must be taken into account that volume of pheromone every artificial and deposits is relative to the nature of solution. This 
algorithm has shown its successful implementation in travelling salesman problem, multi-dimensional knapsack problem and network routing problem etc.[52]. The major characteristics of this algorithm are positive feedback, global search and stability which make its wide application in various fields. Figure. 4 shows stepwise implementation of ACO.

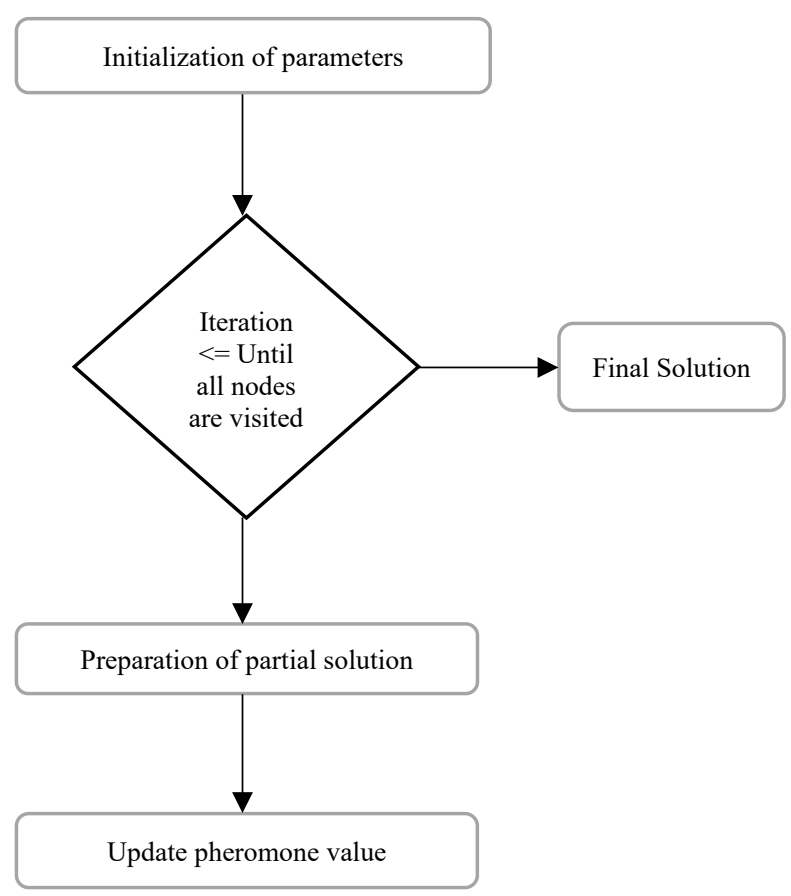

Figure 4. Flowchart of $A C O$

Considering a minimisation problem $(s, f)$ where $s \in S$. Here, $\mathrm{S}$ and $\mathrm{f}$ represents set of viable solutions and objective function respectively. This function allocates a cost value $\mathrm{f}(\mathrm{s})$ to its every solution $\mathrm{s} \in \mathrm{S}$. The major role is to discover the optimal solution $\mathrm{s}^{*}$ of the least cost, where the set of all possible solution is $\mathrm{S}^{*}$. ACO, generally follows two steps to achieve the required solution-

(i) The possible solutions are created using a parameterised probabilistic model.

(ii) The possible results are used to change the model with time so that it intends to bias future sampling towards the least cost solution.

\section{ACO Based Edge Detection}

An ant traverses the problem graph by moving from one pixel to another, however it cannot move to the other pixel in case it is not connected to the pixel, concluding that an ant may not move to its adjacent pixels. The Figure. 5 shows every pixel is related to each pixel which touches its edges or corners.

\begin{tabular}{|c|c|c|}
\hline a-1, b-1 & a, b-1 & a+1, b-1 \\
\hline a-1, b & a, b & $a+1, b$ \\
\hline$a-1, b+1$ & $a, b+1$ & $a+1, b+1$ \\
\hline
\end{tabular}

Figure 5. Connectivity of pixel $(a, b)$

ACO algorithm consists of three major steps namely, Initialization process, Iteration construction \& Update process and Decision process [53].

\section{Initialization Process}

In an image $\mathrm{Mx} \mathrm{N}$, each of the $\mathrm{k}$ ants is designated a random position. Each element in the pheromone matrix is assigned a non-zero constant value $\tau_{\mathrm{i}}$. The heuristic information matrix is designed on changes in the intensity values and is kept constant initially in the first phase [54]. The heuristic value at $(\mathrm{x}, \mathrm{y})$ may be determined by equation $(7)$ :

$$
\eta_{\mathrm{x}, \mathrm{y}}=\frac{V_{c}\left(I_{x, y}\right)}{V_{\max }}
$$

where, $I_{p, q}$ is the pixel intensity at $x, y \cdot V_{c}\left(I_{x, y}\right)$ is only operational on local pixels and is given mathematically by equation (8) $V_{\mathrm{c}}\left(\mathrm{I}_{\mathrm{x}, \mathrm{y}}\right)=\left|\mathrm{I}_{\mathrm{x}-1, \mathrm{y}-1}-\mathrm{I}_{\mathrm{x}+1, \mathrm{y}+1}\right|+\left|\mathrm{I}_{\mathrm{x}-1, \mathrm{y}}-\mathrm{I}_{\mathrm{x}+1, \mathrm{y}}\right|+\mid \mathrm{I}_{\mathrm{x}-1, \mathrm{y}+1}-\mathrm{I}_{\mathrm{x}+1, \mathrm{y}-}$
$1|+| \mathrm{I}_{\mathrm{x}, \mathrm{y}-1}, \mathrm{I}_{\mathrm{x}, \mathrm{y}+1} \mid$

$\mathrm{V}_{\max }$ is the maximum intensity variation in entire image.

\section{Iteration Construction and Update Process}

The possibility of the movement of an ant from any pixel is regulated by probability transition matrix [55]. During $\mathrm{n}^{\text {th }}$ construction step, the $\mathrm{k}^{\text {th }}$ ant moves as per the matrix expressed by equation (9) (10)

$$
\mathrm{P}_{\mathrm{x}, \mathrm{y}(\mathrm{n})}=\frac{\left(\tau_{x y}(n)\right)^{\alpha}\left(\eta_{x y}\right)^{\beta}}{\sum y \epsilon \Omega y\left(\tau_{x y}(n)\right)^{\alpha}\left(\eta_{x y}\right)^{\beta}}
$$

where, $\tau_{x y}^{(n)}$ is the pheromone information value.

$\eta_{x y}$ reflects general pixel data $(\mathrm{x}, \mathrm{y})$ for the journey from node $\mathrm{x}$ to .

$\alpha, \beta$ are the constants surrounding pheromone information and selective data..

$\tau_{x y}(n)$ of an individual ant is updated by equation (10). 


$$
\tau_{x y^{(n)}}=(1-\Psi) \cdot \tau_{x y^{(n-1)}}+\Psi \cdot \tau_{x y^{(0)}}
$$

where $\Psi \in[0,1]$ is the pheromone decay constant which transforms the significance of edges that have already been gone through. After the migration of all the ants, pheromone is updated globally making use of equation (11)

$$
\tau^{(n)}=\left\{\begin{array}{c}
(1-\rho) \cdot \tau^{(n-1)}+\rho \cdot \Delta \tau^{(k)} \text { if }(x, y), \text { best tour } \\
\tau^{(n-1)}, \text { otherwise }
\end{array}\right.
$$

Here, $\rho \in[0,1]$ and $\Delta \tau^{(k)}$ are the evaporation constant and quantity of pheromone obtained by an ant respectively. The pheromone is mathematically given by the equation (12)

\section{Decision Process}

$$
\Delta \tau^{(k)}=\frac{C}{L^{k}}
$$

For a good lower bound on the feasible solution, cost is available. For this following quality function may be used

$Q_{f}=\left(S \mid S_{1}, \ldots, S_{t}\right)=\tau_{\circ}\left(1-\frac{f(s)-L B}{\bar{f}-L B}\right)=\tau_{\circ}\left(\frac{\bar{f}-f(s)}{\bar{f}-L B}\right)$

where, $\mathrm{f}$ and LB are the total cost of the last $\mathrm{k}$ solutions and the lower limit on the cost of the viable solution respectively. With this consistency feature, solutions are measured by comparing their expense, instead of using total cost estimates, with the estimated cost of other recent solutions. Additionally, depending on the similarity of the average cost, the quality function is immediately scaled to the lower bound.

\subsection{Genetic Algorithm}

GAs are effective, flexible and scalable search augmentation techniques driven by evolutionary conventions and usual genetics suggesting parallelism. There are various components of GA including chromosomes, population of chromosome, fitness function, selection, crossover and mutation which follows the given equation 14 .

$W(t+1)=\left\{\begin{array}{c}\left(1 / \alpha_{1}\right) \cdot W(t), \text { if } \overrightarrow{b_{\imath}} \in \text { for all } t-K+1 \leq i \leq t \\ \alpha_{2}, \text { if } \overrightarrow{b_{\imath}} \in S-F \text { for all } t-K+1 \leq i \leq t \\ W(t), \text { otherwise }\end{array}\right.$

Where, $\mathrm{S}$ is the solution set, $\mathrm{F} \subseteq \mathrm{S}$ is a set of all possible solutions, $\overrightarrow{b_{l}}$ is the best chromosome in terms of function evaluation in generation i, $\alpha_{1}, \alpha_{2}>1$ and $\alpha_{1} \neq \alpha_{2}$ (So that cycling is avoided). All the genetic operators are then introduced one after the other in order to produce an advanced breed of chromosomes in which the predicted output of all the chromosomes is higher than the preceding one [56]. Figure 6 presents the flowchart of GA.

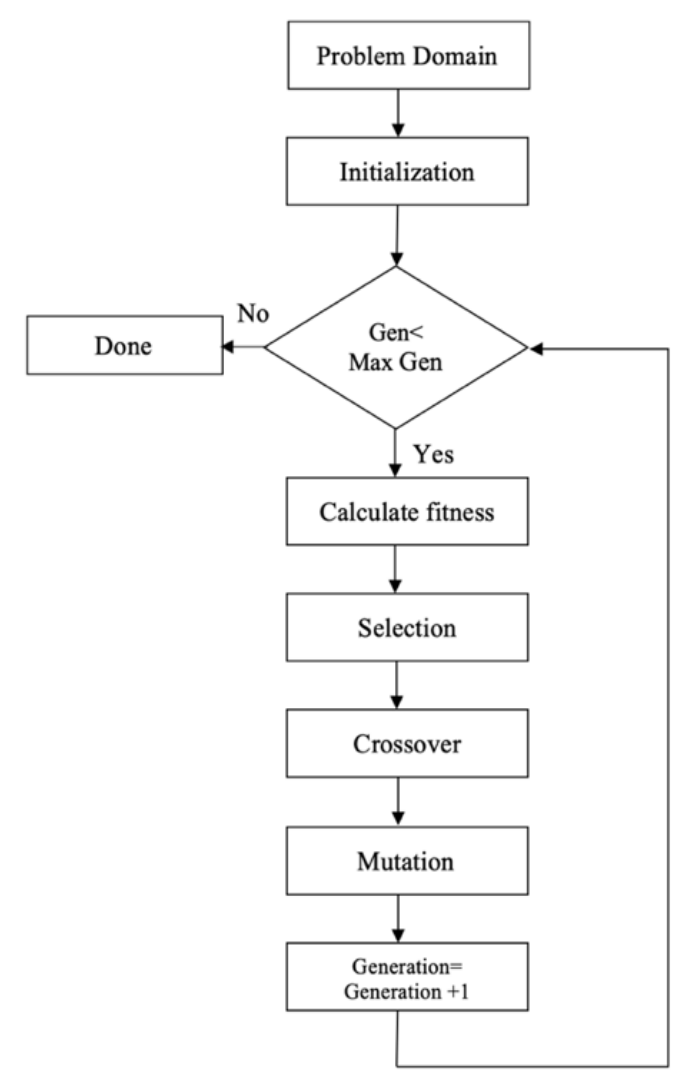

Figure 6. Structure of Genetic Algorithm

The GA follows the following algorithm for successful implementation:

Step 1: Begin with arbitrary generated $\mathrm{N}$ chromosome population, here $\mathrm{N}$ and 1 depict the population number and chromosome x's length respectively.

Step 2: Determine the $\varphi(\mathrm{x})$ feature fitness value of every chromosome $\mathrm{x}$.

Step 3: Repeat until N children are created:

A set of chromosomes is probabilistically derived from the existing population using the fitness function value.

Produce a child using both the operators-crossover and mutation.

Step 4: Substitute an existing population for a newly created one.

Step 5: Go to step 2

GAs are highly promising form of impartial optimization. They are continuously becoming common in image processing. Optimization eminence depends heavily on the selected chromosome encoding system, crossover and mutation techniques, as well as the fitness role [57]. The algorithm allows a rigorous search to be done without trapping local extremes, thus becoming strong alternatives to traditional methods of optimization. 


\subsection{Fuzzy C Means Clustering}

Clustering techniques are constructive database design analysis approaches that have evolved as popular methods for unsupervised pattern recognition and are used in several fields of relevance, including machine learning, data mining, pattern recognition etc [58]. If the algorithm is capable of reducing an error function, the number of classes or clusters is also called C-Means. Although the classes with fuzzy technique are known as Fuzzy C Means. The FCM solution uses a fuzzy membership that designates each class a degree of membership. In a mixture modelling assumption, in fuzzy clustering, the value of the degree of membership is proportional to the probability of pixels. The advantage of FCM is that new clusters are created from data points with similar membership values to current groups [59]. In the FCM method, there are essentially three simple operators: the fuzzy membership function, the partition matrix, and the objective function. These operators may be mathematically summarised as:

$$
\begin{aligned}
& P M_{f c=}\left\{W \in R^{c n} \mid w_{i k}\right. \\
& \in[\mathbf{0}, \mathbf{1}], \forall_{i}, \boldsymbol{k} ; \\
& \sum_{i=1}^{c} w_{i k}=1, \forall k ; 0<\sum_{k=1}^{n} w_{i k}<n, \forall i
\end{aligned}
$$

where, PMfc is the partition matrix, $\mathbf{1} \leq \boldsymbol{i} \leq \boldsymbol{c}, \mathbf{1} \leq \boldsymbol{k} \leq \boldsymbol{n}$.

$$
J_{m}(W, P)=\sum_{\substack{1 \leq k \leq n \\ 0 \leq i \leq c}}\left(w_{i k}\right)^{m}\left(d_{i k}\right)^{2}
$$

where, $\boldsymbol{J}_{\boldsymbol{m}}(\boldsymbol{W}, \boldsymbol{P})$ is the objective function,$\left(\boldsymbol{d}_{\boldsymbol{i} \boldsymbol{k}}\right)=$ $\left\|\boldsymbol{x}_{\boldsymbol{k}}-\boldsymbol{p}_{\boldsymbol{i}}\right\|, \boldsymbol{m} \in(\mathbf{1},+\infty), \boldsymbol{d}_{\boldsymbol{i} \boldsymbol{k}}$ is the Euclidian distance from object $\mathrm{xk}$ to the cluster centre pi.

$$
\mu_{i, j}=\left[\sum_{t=1}^{c}\left(\frac{|| x_{j}-v_{i}|| A}{|| x_{j}-v_{t}|| A}\right) \frac{2}{m-1}\right]-1
$$

where, $\boldsymbol{\mu}_{\boldsymbol{i}, \boldsymbol{j}}$ is the membership value of the jth sample and ith cluster. $\mathrm{c}$ is the number of clusters, $\mathrm{xj}$ is the jth sample and vi cluster centre of the $\mathrm{i}^{\text {th }}$ cluster. \|\| $\mathrm{A}$ is the norm function [60].

FCM is initialization-sensitive and quickly stuck in the local optimum for image segmentation among the fuzzy clustering methods because it has powerful robust uncertainty features and can hold far more data than the tough segmentation method. At each iteration, the clustering is a two-pass operation. To assess the degree of membership in the spatial domain, the first pass is similar in the regular algorithm. The association knowledge of every pixel is mapped with the spatial domain in the secondary pass, and from that, the spatial function is evaluated. The FCM process continues with the most recent membership merged with the spatial objective [61]. If, for two consecutive iterations, the maximum distance between two cluster centres is less than one threshold, the iteration is stopped. Defuzzification is added after convergence to bind all the pixels to certain cluster.

\subsection{Particle Swarm Optimization}

PSO is a global search technique based on swarm intelligence [62]. This is inspired by animals living or travelling in swarm social activities. PSO was proposed in 1995 by Kennedy and Eberhart [63]. The PSO manages a swarm of solutions to the optimization problem being handled. The real number code is also accepted by the PSO whose calculation is simple and the direct solution is easily represented. Each solution for a candidate is referred to as a particle. If there exist $\mathrm{n}$ variables in optimization problem, then every single particle stages an n-dimensional point in the search space. A particle's efficiency or fitness is calculated applying a fitness function. The fitness function checks how similar a particle is to the solution user seeks. All the particles fly through the quest room, changing its location depending upon their distance from their best exclusive location and the distance from the swarm's finest particle. In Figure 7, the flowchart of PSO has been represented. The effectiveness of particles is estimated using a fitness function that reflects the maximization. The following data is preserved by each particle $i$ [64]:

$\mathrm{xi}$, the present position of $\mathrm{i}$; vi, the present velocity of $i$; $\mathrm{yi}$, the best personal position of $\mathrm{i}$.

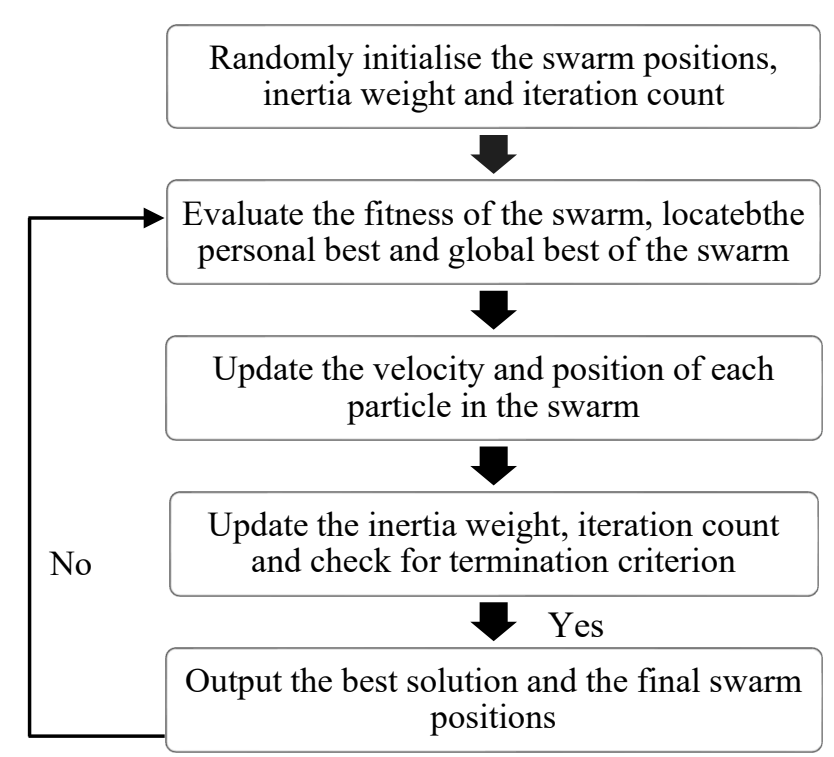

Figure 7. Flowchart of PSO 
Therefore, the strongest personal location is recollection. If $\mathrm{M}$ is the objective function, i's personal best at a time $\tau$ may be updated as:

$$
y_{i}(\tau+1)= \begin{cases}y_{i}(\tau) & \text { if } M\left(x_{i}(\tau+1)\right) \geq M\left(y_{i}(\tau)\right) \\ x_{i}(\tau+1) & \text { if } M\left(x_{i}(\tau+1)\right)<M\left(y_{i}(\tau)\right)\end{cases}
$$

One of PSO's specific concepts is that of the sharing of information between swarm's participants. In order to allow other particles to change their position towards the best, this exchange of knowledge is used to find the best particle(s) or location(s) in the swarm. The best neighbourhood position is specified to globally as the best particle in entire neighbourhood, and the resulting algorithm is denoted as the best PSO [65]. For gbest PSO model, best particle from the whole swarm is calculated as

$$
\hat{y}(\tau) \in\left\{y_{0}, y_{1}, \ldots y_{n}\right\}=\min \left\{M \left(y_{0}(\tau), M\left(y_{1}(\tau), \ldots . . M\left(y_{n}(\tau)\right)\right\}\right.\right.
$$

Here, $n=$ total number of particles in the swarm. The algorithm defined as the lbest PSO where smaller neighbourhoods are considered. Similarly, for lbest PSO, the neighbourhoods are defined as:

$N_{j}=\left\{y_{i-l}(\tau), y_{i-l+1}(\tau), \ldots . y_{i-l}(\tau), y_{i-1}(\tau), y_{i}(\tau), y_{i+1}(\tau), \ldots . y_{i+l-1}(\tau), y_{i+1}(\tau)\right\}$

The best particle in the neighbourhood here, may be calculated as:

$\widehat{y}_{j}(\tau+1) \in N_{j} \mid M\left(\hat{y}_{j}(\tau+1)\right)=\min \left\{M\left(y_{i}\right)\right\}, \forall y_{i} \in N_{j}$

PSO is continuous which require modification while handling the discrete problems. Any values dependent on their existing location in particle space and the resulting velocity vector may be used by the variables in PSO [66]. This algorithm also requires a smaller number of variables, thereby requiring a smaller number of iterations.

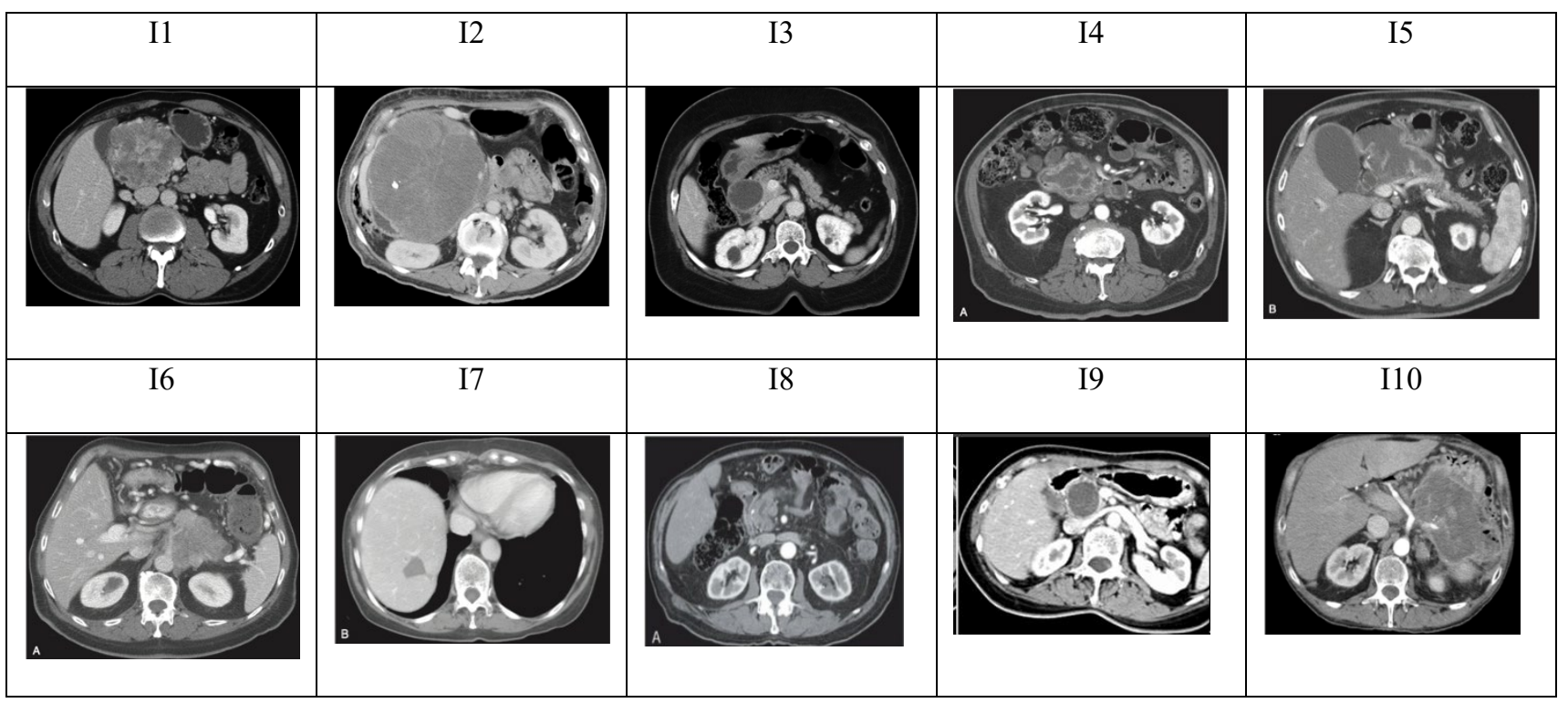

Figure 8. Original CT Images of Pancreatic Tumor 


\section{Results and Discussion}

The CT scans usually contain various irregularities due to deviations in tumorous image. Further, the unwanted noise and poor visualisation affect their visibility qualities. Thus, detection and extraction of relevant information becomes highly challenging. To overcome these problems, four classical edge detection segmentation techniques (Roberts, Sobel, Prewitt and Canny), four artificial intelligence optimized edge detection segmentation techniques (ACORoberts, ACO-Sobel, ACO-Prewitt and ACO-Canny), FCM, GA and PSO have been executed and compared. The resultant images after application of classical image segmentation techniques, ACO optimised classical image segmentation techniques, Fuzzy C Means clustering and artificial intelligence (GA and PSO) techniques have been shown in Figure 9-11 respectively.

It may be noted in Figure 9 that the edges of the image are visible as broken pieces, possibly depicting loss of some important edges. These broken edges are difficult to connect. Edge linking should take into account at least two endpoints. As a result, potential compensable edges from these endpoints must be found. The original image's local details can be explored further to aid in the linking of broken parts and the quest for missing edges.

Figure 10 presents ACO optimised CT images which overcome the concerns of broken edges. To minimise computation load, this method uses four moving policies.
After a finite number of iterations, the remaining pheromone as compensable edges is obtained. Since the proposed redundancy-reduction policies shortens the ant's search route, this approach is efficient and gives better edge visualisation as compared to the classical edge detection.

Figure 11 shows the resultant images after implementation of FCM, GA and PSO.

It is seen clearly that the output images obtained using PSO are visually enhanced as compared to both FCM and GA.

The minute details of the human anatomy in CT can be easily understood and diagnosed with the images obtained from PSO. Also, due to adjustment of only few

parameters, this technique is cheaper as well as consumes less computational time.

To evaluate the performance quantitatively, entropy has been determined for test images. Image entropy indicates the amount of information stored in an image. The low entropy images are dark in texture and have no difference with the adjacent pixels [67-68]. The entropy values [69] of test images after application of classical Image segmentation techniques, ACO Optimized classical image segmentation techniques and for Fuzzy $\mathrm{C}$ means clustering and artificial intelligence (GA and PSO) techniques are presented in Table $1-3$.

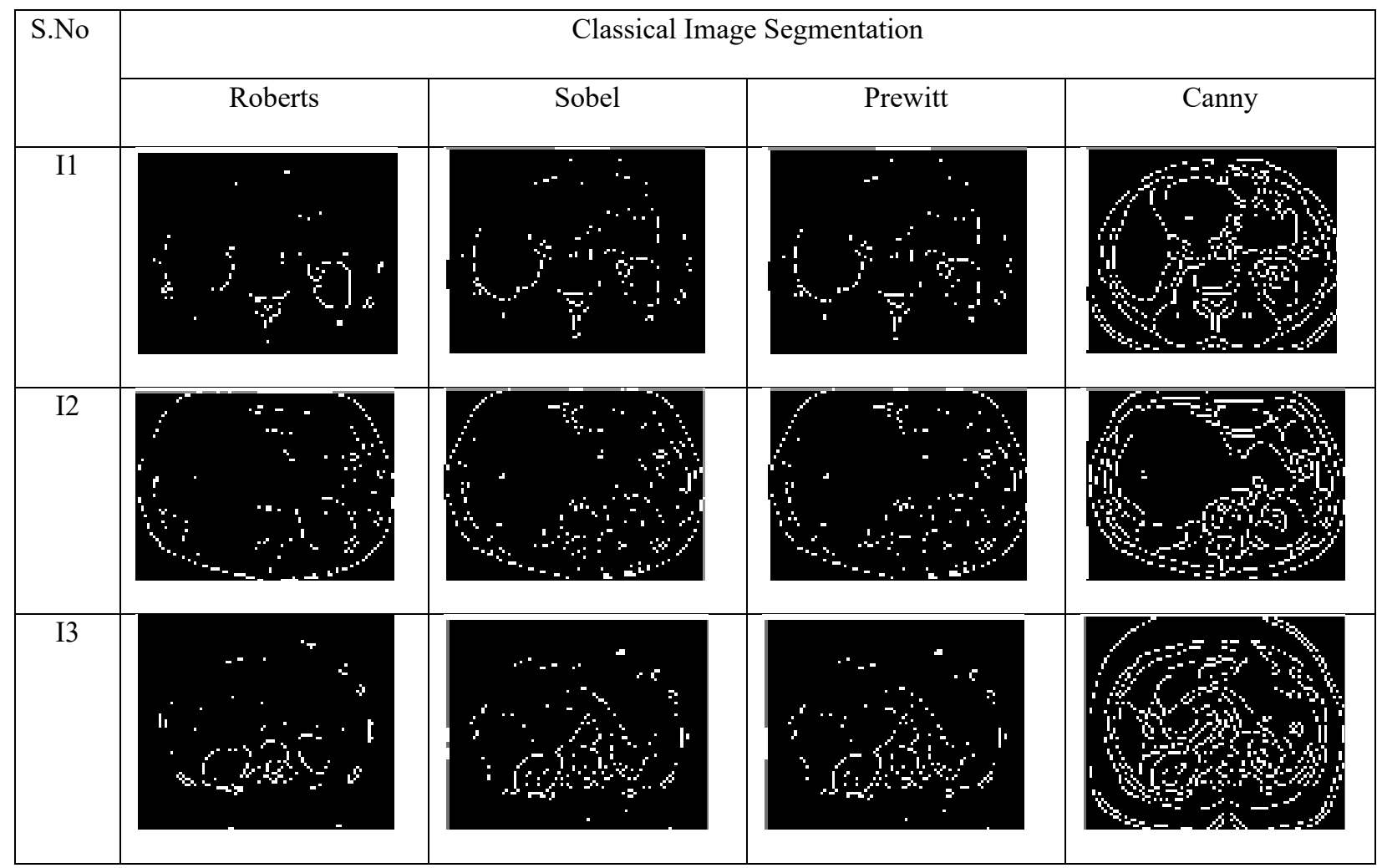




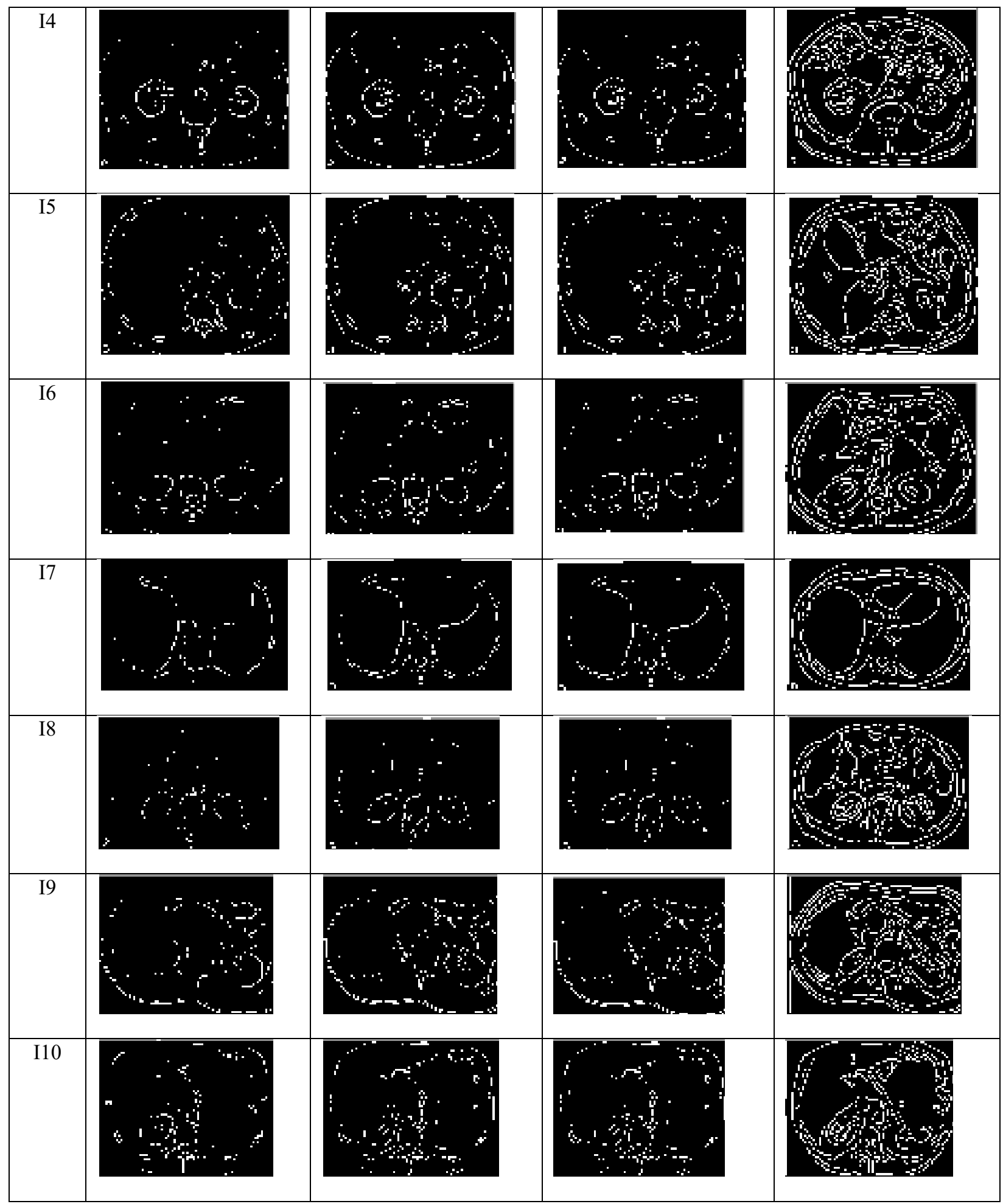

Figure 9. CT Images after Classical Image Segmentation (Prewitt, Roberts, Sobel and Canny Edge Detection) Techniques

\begin{tabular}{|l|l|}
\hline S.No & ACO Optimized Classical Image Segmentation \\
\hline
\end{tabular}




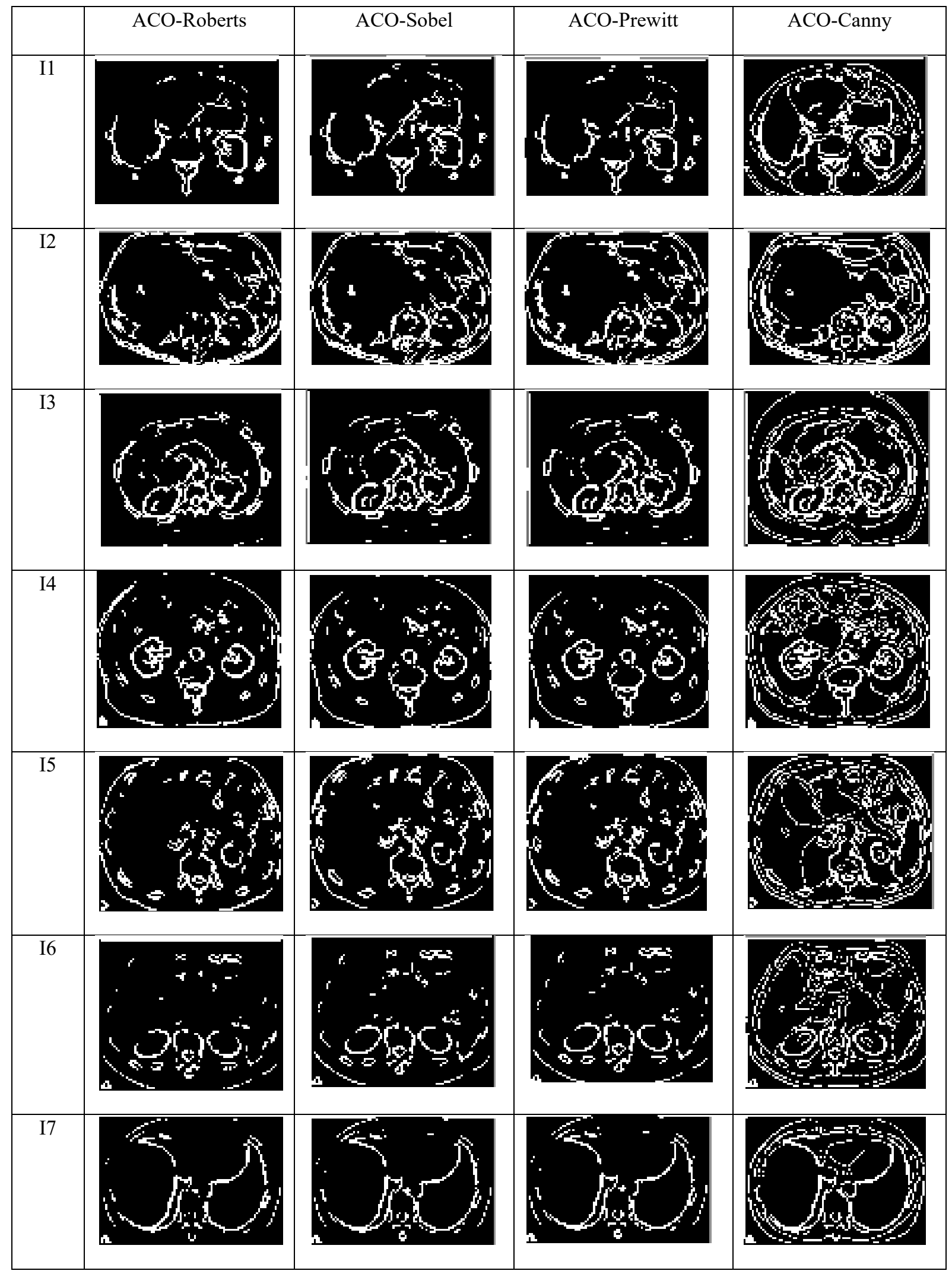




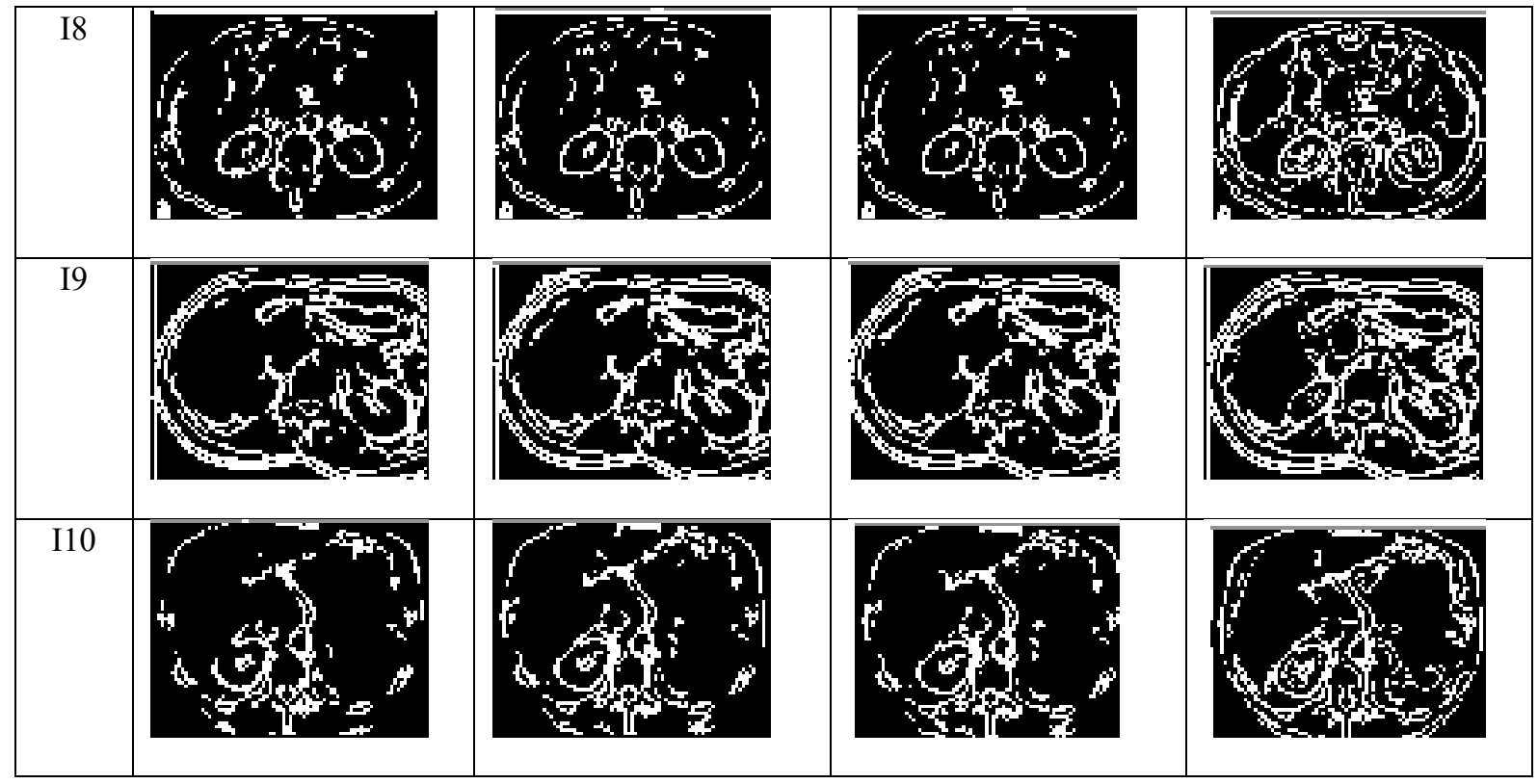

Figure 10. Resultant CT Images after ACO Optimized Classical Image Segmentation (ACO-Roberts, ACO-Sobel, ACO-Prewitt and ACO-Canny Edge Detection) Techniques

\begin{tabular}{|c|c|c|c|}
\hline \multirow[t]{2}{*}{ S.No } & \multirow{2}{*}{$\begin{array}{l}\text { Fuzzy C Means } \\
\text { Clustering (FCM) }\end{array}$} & \multicolumn{2}{|c|}{ Artificial Intelligence Techniques } \\
\hline & & $\begin{array}{c}\text { Genetic Algorithm } \\
\text { (GA) }\end{array}$ & $\begin{array}{c}\text { Particle Swarm } \\
\text { Optimization (PSO) }\end{array}$ \\
\hline I1 & & & \\
\hline I 2 & & & \\
\hline $\mathrm{I} 3$ & & & \\
\hline I4 & & & \\
\hline & & & \\
\hline & & & \\
\hline
\end{tabular}




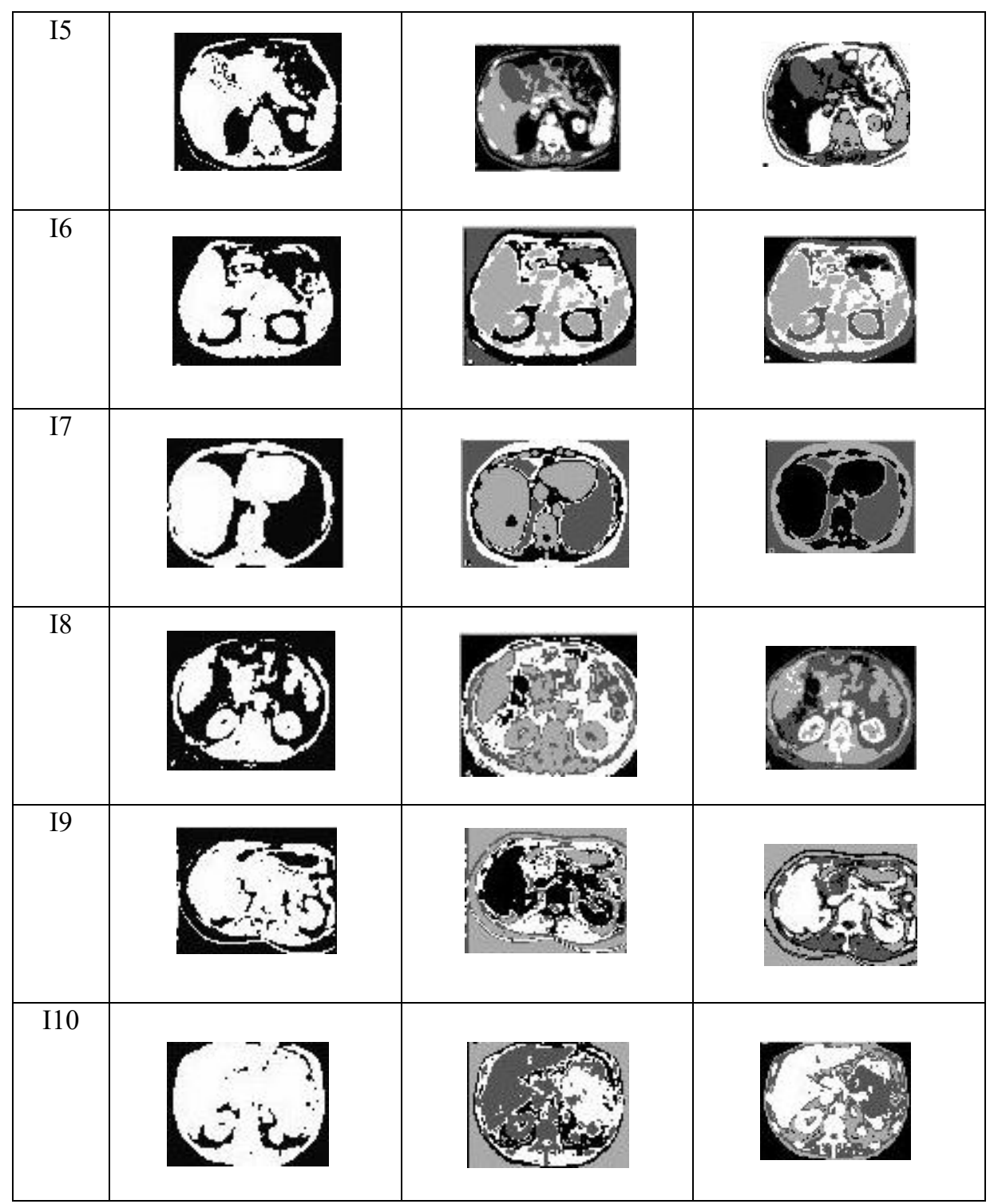

Figure 11. Resultant CT Images after Fuzzy C Means Clustering and Artificial Intelligence (GA and PSO) Techniques

For test images I 1-I 10, the entropy values obtained with Roberts edge detection segmentation are 0.2067, 0.2575, $0.2098 \ldots$ and 0.2413 respectively. The entropy values obtained with Sobel edge detection segmentation images are $0.2271,0.3075,0.2253 \ldots$ and 0.2065 respectively. The values computed with Prewitt edge detection segmentation are $0.2247,0.3006,0.2228 \ldots$ and 0.2551 respectively. Similarly, the values computed after Canny are 0.5308 , $0.5233,0.5726 \ldots$ and 0.5391 respectively. Further, with application of ACO technique, the improved values of entropy are obtained. The ACO-Roberts yields in entropy values- $0.4679,0.6506,0.5268 \ldots$ and 0.5631 respectively. The entropy values obtained with ACO-Sobel are 0.5194,
$0.6778,0.5103 \ldots$ and 0.5900 respectively. The entropy values computed after ACO-Prewitt are 0.4731, 0.6747, $0.5106 \ldots$ and 05902 respectively. Similarly, the entropy values obtained after ACO-Canny are 0.7155, 0.7438, $0.7412 \ldots$ and 0.7047 respectively. From Fig. 8, with implementation of FCM, the resultant image's entropy values are $3.9736,3.8547,4.2755,4.1701 \ldots$ and 3.7274 respectively. With GA, the improved entropy values obtained are 5.0216, 4.5864, 4.5631, 4.6198...... and 4.8516 respectively. With $\mathrm{PSO}$, the remarkably improved entropy values are obtained. These values for respective images are 5.1608, 4.8167, 5.1025, 4.9439....and 4.8795 respectively. The comparative analysis of simulation 
results for entropy values is also carried out pictorially by column chart as depicted in Figure 12-14. The highest values of entropy are obtained with PSO algorithm. This indicates the superior quality of resultant CT images in comparison with all other techniques i.e. classical edge detection segmentation (Prewitt, Roberts, Sobel and Canny), ACO-edge detection segmentation (ACO-Prewitt, ACO-Roberts and ACO-Sobel), FCM and GA techniques. This ensures the far superior visibility aspects of output medical images referring to more information.

Table 1. Entropy for Classical Image Segmentation Techniques

\begin{tabular}{|c|c|c|c|c|}
\hline \multirow[t]{3}{*}{ Image } & \multicolumn{4}{|c|}{ Entropy } \\
\hline & \multicolumn{4}{|c|}{ Classical Image Segmentation } \\
\hline & Roberts & Sobel & Prewitt & Canny \\
\hline I1 & 0.2067 & 0.2271 & 0.2247 & 0.5308 \\
\hline $\mathrm{I} 2$ & 0.2575 & 0.3075 & 0.3006 & 0.5233 \\
\hline I3 & 0.2098 & 0.2253 & 0.2228 & 0.5726 \\
\hline I4 & 0.2308 & 0.2561 & 0.2484 & 0.6009 \\
\hline I5 & 0.2268 & 0.2613 & 0.2569 & 0.5638 \\
\hline I6 & 0.2002 & 0.2415 & 0.2357 & 0.5596 \\
\hline I7 & 0.2211 & 0.2526 & 0.2501 & 0.4750 \\
\hline I8 & 0.1867 & 0.2297 & 0.2264 & 0.5808 \\
\hline I9 & 0.2398 & 0.3052 & 0.2979 & 0.6334 \\
\hline I10 & 0.2413 & 0.2605 & 0.2551 & 0.5391 \\
\hline Average & 0.2220 & 0.2566 & 0.2518 & 0.5579 \\
\hline
\end{tabular}

Table 2. Entropy for ACO Optimised Classical Image Segmentation Techniques

\begin{tabular}{cc}
\hline Image & Entropy \\
ACO- Optimised Classical Image \\
Segmentation
\end{tabular}

\begin{tabular}{ccccc}
\hline & ACO- & ACO- & ACO- & ACO- \\
& Roberts & Sobel & Prewitt & Canny \\
\hline I1 & 0.4679 & 0.5194 & 0.4731 & 0.7155 \\
\hline I2 & 0.6506 & 0.6778 & 0.6747 & 0.7438 \\
\hline I3 & 0.5268 & 0.5103 & 0.5106 & 0.7412 \\
\hline I4 & 0.5566 & 0.5350 & 0.5313 & 0.7512 \\
\hline I5 & 0.5584 & 0.5812 & 0.5789 & 0.7167 \\
\hline I6 & 0.4240 & 0.4580 & 0.4531 & 0.6806 \\
\hline I7 & 0.5216 & 0.5074 & 0.5085 & 0.6522 \\
\hline I8 & 0.5628 & 0.5770 & 0.5735 & 0.7047 \\
\hline I9 & 0.8354 & 0.8403 & 0.8377 & 0.8972 \\
\hline I10 & 0.5631 & 0.5900 & 0.5902 & 0.7047 \\
\hline Average & 0.5667 & 0.5796 & 0.5731 & 0.7307 \\
\hline & & & & \\
\hline
\end{tabular}

Table 3. Entropy for FCM, GA and PSO

\begin{tabular}{|c|c|c|c|}
\hline \multirow[t]{4}{*}{ Image } & \multicolumn{3}{|c|}{ Entropy } \\
\hline & \multirow[t]{3}{*}{ FCM } & \multirow{2}{*}{\multicolumn{2}{|c|}{$\begin{array}{c}\text { Artificial Intelligence } \\
\text { Techniques }\end{array}$}} \\
\hline & & & \\
\hline & & GA & PSO \\
\hline I1 & 3.9736 & 5.0216 & 5.1608 \\
\hline $\mathrm{I} 2$ & 3.8547 & 4.5864 & 4.8167 \\
\hline I3 & 4.2755 & 4.5631 & 5.1025 \\
\hline $\mathrm{I} 4$ & 4.1701 & 4.6198 & 4.9439 \\
\hline I5 & 4.2006 & 4.4288 & 4.8885 \\
\hline I6 & 4.1971 & 4.8012 & 4.8982 \\
\hline I7 & 4.2217 & 4.5239 & 4.5810 \\
\hline I8 & 4.2066 & 4.7960 & 4.8224 \\
\hline I9 & 4.2911 & 4.7713 & 4.9882 \\
\hline
\end{tabular}




\begin{tabular}{cccc}
\hline I10 & 3.7274 & 4.8516 & 4.8795 \\
\hline Average & 4.1118 & 4.6963 & 4.9081 \\
\hline
\end{tabular}

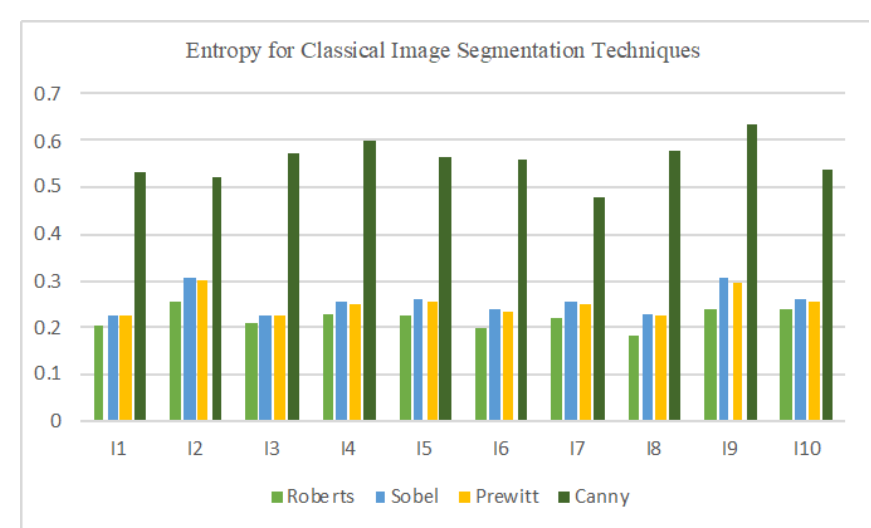

Figure 12. Entropy for Classical Image Segmentation Techniques

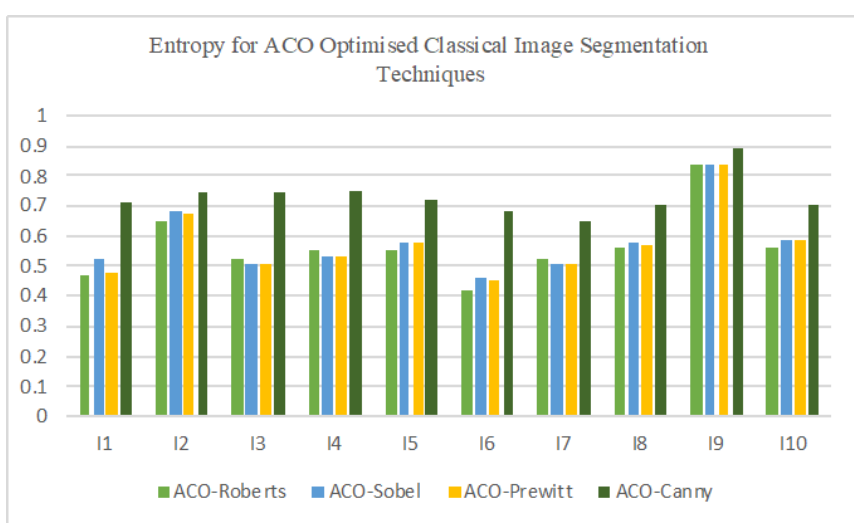

Figure 13. Entropy for ACO Optimised Classical Image Segmentation Techniques

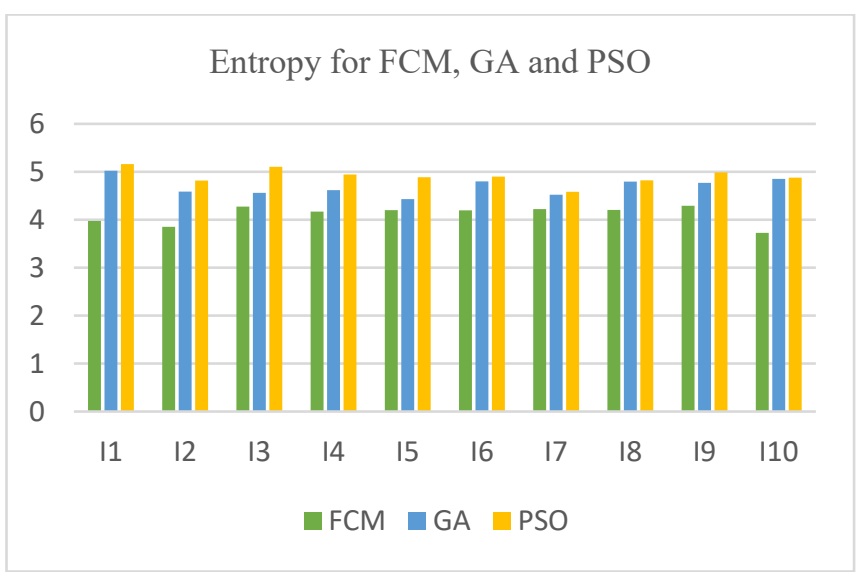

Figure 14. Entropy for FCM, GA and PSO

\section{Conclusion}

The precise interpretation of complex $\mathrm{CT}$ images is crucial for accurate diagnosis and early detection of pancreatic tumor. In this work, the comparison of simulation results has been carried out by using image quality parameter i.e. entropy. The entropy value obtained for classical segmentation methods- Roberts, Sobel ,Prewitt and canny is least when compared with others. Although the values obtained using ACO methods give improved results when compared with classical methods. However, analysis of entropy value for FCM, GA and PSO conclude that PSO technique provides considerably improved results for performance evaluation parameter ensuring far better visibility qualities of output images. The highly optimistic results may be utilized for correct diagnosis and early detection of pancreatic tumors using segmented CT images. This may further help in accurate and appropriate disease management with availability of crucial information to decide the resection margins while operating on a patient. Further, this might become the key instrument in salvaging as much normal parenchyma as possible and lead to least possible chances of morbidity with maximum chances of survival.

Declaration: This research did not receive any specific grant from funding agencies in the public, commercial, or not-for-profit sectors.

\section{References}

[1] Yan Z, Ma C, Mo J, Han W, Lv X, Chen C, Chen C, Nie X. Rapid identification of benign and malignant pancreatic tumors using serum Raman spectroscopy combined with classification algorithms. Optik. 2020 Apr 1;208:164473.

[2] Rogowska J. Overview and fundamentals of medical image segmentation. Handbook of medical imaging, processing and analysis. 2000 Oct 15:69-85. 
[3] Maulik U. Medical image segmentation using genetic algorithms. IEEE Transactions on information technology in biomedicine. 2009 Mar;13(2):166-73.

[4] N. Mittal, S. Tanwar and S. K. Khatri. Identification \& enhancement of different skin lesion images by segmentation techniques. IEEE International Conference on Reliability, Infocom Technologies and Optimization. 609614, 2017

[5] Smistad E, Falch TL, Bozorgi M, Elster AC, Lindseth F. Medical image segmentation on GPUs-A comprehensive review. Medical image analysis. 2015 Feb 1;20(1):1-8.

[6] Taha AA, Hanbury A. Metrics for evaluating 3D medical image segmentation: analysis, selection, and tool. BMC medical imaging. 2015 Dec;15(1):29.

[7] S. Sengupta and N. Mittal. Analysis of various techniques of feature extraction on skin lesion images. IEEE International Conference on Reliability, Infocom Technologies and Optimization. India. 651-656. 2017.

[8] Zhang B, Sun X, Gao L, Yang L. Endmember extraction of hyperspectral remote sensing images based on the ant colony optimization (ACO) algorithm. IEEE transactions on geoscience and remote sensing. $2011 \mathrm{Jul}$;9(7):2635-46.

[9] Dorigo M, Stutzle T. A short convergence proof for a class of ACO algorithms. IEEE Transactions on Evolutionary Computation. 2002;6.

[10] N. Mittal, H. P. Singh and R. Gupta. Decomposition \& reconstruction of medical images in MATLAB using different Wavelet parameters. 2015 International Conference on Futuristic Trends on Computational Analysis and Knowledge Management (ABLAZE), India. 647-653 2015

[11] Alomoush W, Alrosan A, Norwawi N, Alomari Y, Albashish D, Almomani A, Alqahtani M. A survey: Challenges of image segmentation based fuzzy c-means clustering algorithm. Journal of Theoretical and Applied Information Technology. 2018.

[12] Dubey YK, Mushrif MM. FCM clustering algorithms for segmentation of brain MR images. Advances in Fuzzy Systems. 2016 Mar 1;2016.

[13] Binitha S, Sathya SS. A survey of bio inspired optimization algorithms. International journal of soft computing and engineering. 2012 May;2(2):137-51.

[14] Sudhriti Sengupta, Neetu Mittal \& Megha Modi. Improved skin lesions detection using color space and artificial intelligence techniques, Journal of Dermatological Treatment, 31:5. 511-518. 2019

[15] $\mathrm{Yu} \mathrm{M}$. Image segmentation using genetic algorithm and morphological operations.

[16] Katoch S, Chauhan SS, Kumar V. A review on genetic algorithm: past, present, and future. Multimedia Tools and Applications. 2020 Oct 31:1-36

[17] Le Hégarat-Mascle S, Kallel A, Descombes X. Application of ant colony optimization to image classification using a Markov model with non-stationary neighborhoods. In Image and Signal Processing for Remote Sensing XI 2005 Oct 18 (Vol. 5982, p. 59820C). International Society for Optics and Photonics.

[18] Dhruv B., Mittal N., Modi M. Comparative Analysis of Edge Detection Techniques for Medical Images of Different Body Parts. In: Panda B., Sharma S., Roy N. (eds) Data Science and Analytics. Communications in Computer and Information Science, 799. 2018

[19] Dorigo, M., Maniezzo, V. and Colorni, A., 1996. Ant system: optimization by a colony of cooperating agents. IEEE Transactions on Systems, man, and cybernetics, Part B: Cybernetics, 26(1), pp.29-41.
[20] Khanna K, Madan Arora S. Ant colony optimization towards image processing. Indian J Sci Technol. 2016 Dec;9(48):1-9.

[21] Wang G. A comparative study of cuckoo algorithm and ant colony algorithm in optimal path problems. InMATEC Web of Conferences 2018 (Vol. 232, p. 03003). EDP Sciences.

[22] Aher CN and Jena AK. Soft Computing based Approaches for Classifying Diseases using Medical Diagnosis Dataset. IEEE International Conference on Emerging Smart Computing and Informatics 2020 Mar 12 (pp. 77-81).

[23] Kaur P and Sharma M. Analysis of data mining and soft computing techniques in prospecting diabetes disorder in human beings: a review. Int. J. Pharm. Sci. Res. 2018 Jul 1;9:2700-19.

[24] Sharma S, Singh G and Sharma M. A Comprehensive Review and Analysis of Supervised-Learning and Soft Computing Techniques for Stress Diagnosis in Humans. Computers in Biology and Medicine. 2021 May 5:104450.

[25] Sharma M and Romero N. Future prospective of soft computing techniques in psychiatric disorder diagnosis. EAI Endorsed Transactions on Pervasive Health and Technology. 2018 Jul 1;4(15).

[26] Sharma S and Singh G. Diagnosis of cardiac arrhythmia using Swarm-intelligence based Metaheuristic Techniques: A comparative analysis. EAI Endorsed Transactions on Pervasive Health and Technology. 2020;6(23).

[27] Singh R. Nature Inspired Based Meta-heuristic Techniques for Global Applications. International Journal of Computer Applications \& Information Technology. 2020;12(1):303-9.

[28] Bashir ZA, El-Hawary ME. Applying wavelets to shortterm load forecasting using PSO-based neural networks. IEEE transactions on power systems. 2009 Jan 13;24(1):207.

[29] Selvi V, Umarani R. Comparative analysis of ant colony and particle swarm optimization techniques. International Journal of Computer Applications. 2010 Aug;5(4):1-6.

[30] Felzenszwalb PF, Huttenlocher DP. Efficient graph-based image segmentation. International journal of computer vision. 2004 Sep 1;59(2):167-81

[31] Sharma N, Aggarwal LM. Automated medical image segmentation techniques. Journal of medical physics/Association of Medical Physicists of India. 2010 Jan;35(1):3.

[32] He L, Peng Z, Everding B, Wang X, Han CY, Weiss KL, Wee WG. A comparative study of deformable contour methods on medical image segmentation. Image and vision computing. 2008 Feb 1;26(2):141-63.

[33] Pham DL, Xu C, Prince JL. Current methods in medical image segmentation. Annual review of biomedical engineering. 2000 Aug;2(1):315-37.

[34] Droske M, Meyer B, Rumpf M, Schaller C. An adaptive level set method for medical image segmentation. In Biennial International Conference on Information Processing in Medical Imaging 2001 Jun 18 (pp. 416-422). Springer, Berlin, Heidelberg.

[35] Nadernejad E, Sharifzadeh S, Hassanpour H. Edge detection techniques: Evaluations and comparisons. Applied Mathematical Sciences. 2008 Jan;2(31):1507-20.

[36] Sharifi M, Fathy M, Mahmoudi MT. A classified and comparative study of edge detection algorithms. In Proceedings. International conference on information technology: Coding and computing 2002 Apr 8 (pp. 117120). IEEE.

[37] Basu M. Gaussian-based edge-detection methods-a survey. IEEE Transactions on Systems, Man, and Cybernetics, Part C (Applications and Reviews). 2002 Aug;32(3):252-60. 
[38] Perona P, Malik J. Scale-space and edge detection using anisotropic diffusion. IEEE Transactions on pattern analysis and machine intelligence. $1990 \mathrm{Jul} ; 12$ (7):629-39.

[39] Lindeberg T. Edge detection and ridge detection with automatic scale selection. International journal of computer vision. 1998 Nov 1;30(2):117-56.

[40] Maini R, Aggarwal H. Study and comparison of various image edge detection techniques. International journal of image processing (IJIP). 2008;3(1).

[41] Hou Z, Koh TS. Robust edge detection. Pattern Recognition. 2003 Sep 1;36(9):2083-91.

[42] Somkantha K, Theera-Umpon N, Auephanwiriyakul S. Boundary detection in medical images using edge following algorithm based on intensity gradient and texture gradient features.

[43] Senthilkumaran N, Rajesh R. Edge detection techniques for image segmentation-a survey of soft computing approaches. International journal of recent trends in engineering. 2009 May 1;1(2):250.

[44] Bao P, Zhang L, Wu X. Canny edge detection enhancement by scale multiplication. IEEE transactions on pattern analysis and machine intelligence. 2005 Sep;27(9):1485-90.

[45] Pavlidis T, Liow YT. Integrating region growing and edge detection. IEEE Transactions on Pattern Analysis and Machine Intelligence. 1990 Mar;12(3):225-33.

[46] Dorigo M, Birattari M. Ant colony optimization. Springer US; 2010.

[47] Dorigo M, Blum C. Ant colony optimization theory: A survey. Theoretical computer science. 2005 Nov 17;344(23):243-78.

[48] Socha K. ACO for continuous and mixed-variable optimization. In International Workshop on Ant Colony Optimization and Swarm Intelligence 2004 Sep 5 (pp. 2536). Springer, Berlin, Heidelberg.

[49] Merkle D, Middendorf M, Schmeck H. Ant colony optimization for resource-constrained project scheduling. IEEE transactions on evolutionary computation. 2002 Aug;6(4):333-46.

[50] Gambardella LM, Dorigo M. An ant colony system hybridized with a new local search for the sequential ordering problem. INFORMS Journal on Computing. 2000 Aug;12(3):237-55.

[51] Blum C, Roli A. Metaheuristics in combinatorial optimization: Overview and conceptual comparison. ACM computing surveys (CSUR). 2003 Sep 1;35(3):268-308.

[52] Liu XP, Li X, He JQ, Ai B, Peng XJ. Classification of remote sensing images based on ant colony optimization. Journal of remote sensing-Beijing-. 2008;12(2):262.

[53] Song Q, Guo P, Jia Y. Ant colony optimization algorithm for remote sensing image classification using combined features. In2008 International Conference on Machine Learning and Cybernetics 2008 Jul 12 (Vol. 6, pp. 34783483). IEEE.

[54] Dai Q, Liu J. Application of ant colony optimization (ACO) algorithm to remote sensing image classification. In MIPPR 2007: Pattern Recognition and Computer Vision 2007 Nov 15 (Vol. 6788, p. 67881A). International Society for Optics and Photonics.

[55] Brignone M, Bozza G, Randazzo A, Piana M, Pastorino M. A hybrid approach to 3D microwave imaging by using linear sampling and ACO. IEEE Transactions on Antennas and Propagation. 2008 Oct;56(10):3224-32.

[56] Paulinas M, Ušinskas A. A survey of genetic algorithms applications for image enhancement and segmentation. Information Technology and control. 2007 Sep 28;36(3).
[57] Tang L, Tian L, Steward BL. Color image segmentation with genetic algorithm for in-field weed sensing. Transactions of the ASAE. 2000;43(4):1019.

[58] Nayak J, Naik B, Behera H. Fuzzy C-means (FCM) clustering algorithm: a decade review from 2000 to 2014. Computational intelligence in data mining-volume 2 . 2015:133-49.

[59] Izakian H, Abraham A, Snášel V. Fuzzy clustering using hybrid fuzzy c-means and fuzzy particle swarm optimization. In2009 World Congress on Nature \& Biologically Inspired Computing (NaBIC) 2009 Dec 9 (pp. 1690-1694). IEEE.

[60] Yang Y, Huang S. Image segmentation by fuzzy c-means clustering algorithm with a novel penalty term. Computing and informatics. 2012 Jan 27;26(1):17-31.

[61] Chuang KS, Tzeng HL, Chen S, Wu J, Chen TJ. Fuzzy cmeans clustering with spatial information for image segmentation. computerized medical imaging and graphics. 2006 Jan 1;30(1):9-15.

[62] Acharya UK, Kumar S. Particle swarm optimized texture based histogram equalization (PSOTHE) for MRI brain image enhancement. Optik. 2020 Dec 1;224:165760.

[63] Ait-Aoudia S, Guerrout EH, Mahiou R. Medical image segmentation using particle swarm optimization. In2014 18th International Conference on Information Visualisation 2014 Jul 16 (pp. 287-291). IEEE.

[64] Mahalakshmi S, Velmurugan T. Detection of brain tumor by particle swarm optimization using image segmentation. Indian Journal of Science and Technology. 2015 Sep $1 ; 8(22): 1$.

[65] Omran M, Engelbrecht AP, Salman A. Particle swarm optimization method for image clustering. International Journal of Pattern Recognition and Artificial Intelligence. 2005 May; 19(03):297-321.

[66] Omran MG, Salman A, Engelbrecht AP. Dynamic clustering using particle swarm optimization with application in image segmentation. Pattern Analysis and Applications. 2006;1;8(4):332.

[67] Rallabandi VS, Roy PK. Magnetic resonance image enhancement using stochastic resonance in Fourier domain", Magnetic Resonance Imaging 2010. 1;28(9):1361-73

[68] Mittal N. Automatic Contrast Enhancement of Low Contrast Images using MATLAB. International Journal of Advanced Research in Computer Science.2012; 3 (1): 333338.

[69] Schavemaker JG, Reinders MJ, Gerbrands JJ, Backer E. Image sharpening by morphological filtering. Pattern Recognition. 2000;33(6):997-1012. 